\title{
Opinion
}

\section{Why are Patients Reluctant to View Exercise as a Treatment Plan?}

\author{
Mike Swoboda, DHSc, CSCS, EP-C, Exercise is Medicine Credential Level II, FNS" \\ Department of Fitness and Wellness, College of Education and Health Professions, Park University, 92WVaughn Ave, Gilbert, AZ 85233, USA
}

"Corresponding author

Mike Swoboda, DHSc, CSCS, EP-C, Exercise is Medicine Credential Level II, FNS

Assistant Professor, Department of Fitness and Wellness, College of Education and Health Professions, Park University, 92WVaughn Avee, Gilbert, AZ85233, USA; Tel. 480-923-0028 ×3028; E-mail: mswoboda@park.edu

\section{Article information}

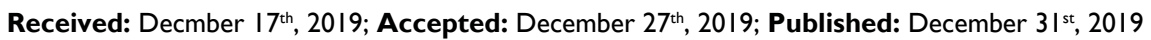

\section{Cite this article}

Swoboda M. Why are patients reluctant to view exercise as a treatment plan? Obes Res Open J. 2019; 6(2): 35-36. doi: I0.17/40/OROJ-6-I40

\begin{abstract}
A healthcare costs continue to rise worldwide, as well as cases of overweight and obese individuals, a pedagogical shift must be made towards a greater use of preventative care and exercise as treatment methods for disease management and prevention. The physical benefits of exercise are not disputed, but costs, proper exercise prescription, and program compliance remain barriers to widespread adoption of exercise as treatment for disease management and improving quality of life. Regular weekly exercise not only improves quality of life in overweight and obese individuals $^{1}$ and individuals with cardiorespiratory illness or disease, ${ }^{2}$ but will also save on healthcare costs. Research has indicated seniors (age <65-years) who regularly participate in an organized exercise program spend on average $\$ 1186-2144$ less than their less active or sedentary peers. ${ }^{3}$ However, the Centers for Disease Control $(\mathrm{CDC})^{4}$ noted that only $22.9 \%$ of Americans achieved the weekly exercise recommendations of strength training two times per week, and at least 150-minutes of moderate-to-vigorous cardiovascular activity weekly.
\end{abstract}

With mounting positive evidence that regular exercise should be a part of all people's lives, why is there a lack of exercise by individuals worldwide? This answer appears to be twofold, and the main determining interaction is between the patient and their primary care physician (PCP).

One such issue is a general expectation of patients to be treated with medication by their PCP for their chief complaints of illness or chronic condition. While nations are starting to adopt policies to reduce the prescription of excessive medications and medical testing, many patients still demand medications for treatment, even when they are not indicated.

Still, the bigger issue exists in PCPs who are not able to properly prescribe exercise. Many PCPs believe that proper exer- cise prescription is simply telling patients to "exercise more," and are unable to differentiate between exercise and physical activity. This extends into discontinuation of exercise as part of an ongoing disease management treatment plan, such as in the case of osteoarthritis. When exercise is prescribed as part of an ongoing maintenance plan (as opposed to a short-term solution), noncompliance and complete discontinuation of exercise programs becomes inevitable for the majority of participants.

There is no simple solution to this problem, but steps can be put in place to start to curb the acceptance of using exercise as a long-term preventative treatment method by at-risk patients. Physicians without an in-house exercise specialist should partner with organizations, universities, or individuals within the community to provide access to adequate exercise prescription to their patients. Allowing a fitness professional to provide an exercise program to a patient that fits their ability level and access to services, overall understanding of how to exercise will increase. Without direction, or by simply telling a patient to exercise without specific instructors, sedentary behaviors will continue to rise. Changing patient's perceptions to adopting exercise as a treatment plan will ultimately be the more trying issue. A paradigm shift of viewing exercise as something temporary to recover from injury or to be a short-term treatment needs to change to a lifelong endeavor. PCPs who are able to convey the value of exercise as a type of medicine, rather than something that should just be part of a patient's life without a specific reason, will ultimately have the most success with longterm patient compliance. Although intrinsic motivation is the most powerful form of motivation to elicit behavior change, it may be necessary for healthcare providers and employers to use extrinsic motivators to begin the process of behavior change. This could potentially include financial incentives and discounts on healthcare costs when a patient stays compliant with an exercise program. Incorporating the exercise professional into the healthcare sys- 
tem by allowing insurance reimbursements and billing is one of the biggest hurdles to achieving this goal. Ultimately, there must be a ubiquitous acceptance of exercise being part of the field of healthcare and medicine for the long-term reversal of obesity and obesity-related illnesses.

\section{REFERENCES}

1. Wu J, Davis-Ajami ML, Lu ZK. Real-world impact of ongoing regular exercise in overweight and obese US adults with diabetes on health care utilization and expenses. Prim Care Diabetes. 2019; 13(5): 430-440. doi: 10.1016/j.pcd.2019.02.003

2. Grootjans-van Kampen I, Engelfriet PM, van Baal PHM. Disease prevention: Saving lives or reducing health care costs? PLoS One. 2014; 9(8): 1-5. doi: 10.1371/journal.pone.0104469

3. Crossman AF. Healthcare cost savings over a one-year period for silver sneakers group exercise participants. Health Behavior \& Policy Review. 2018;5(1):40-46. doi: 10.14485/HBPR.5.1.4
4. Blackwell DL, Clarke, TC. State Variation in Meeting the 2008 Federal Guidelines for both aerobic and muscle-strengthening activities through leisure-time physical activity among adults aged 1864: United States, 2010-2015. Natl Health Stat Report. 2018; (112): $1-22$.

5. Malhotra A, Maughan D, Ansell J, et al. Choosing Wisely in the UK: The Academy of Medical Royal Colleges' initiative to reduce the harms of too much medicine. BMJ. 2015; 350: h2308. doi: 10.1136/bmj.h2308

6. Seth A. Exercise prescription: what does it mean for primary care? Br J Gen Pract. 2014; 64(618): 12-13. doi: 10.3399/bjgp14X676294

7. Campbell R, Evans M, Tucker M, Quilty B, Dieppe P, Donovan JL. Why don't patients do their exercises? Understanding non-compliance with physiotherapy in patients with osteoarthritis of the knee. J Epidemiol Community Health. 2001; 55(2): 132. doi: 10.1136/jech.55.2.132 